\title{
A survey of adult patients with inflammatory bowel disease to determine their awareness of nutritional treatments and access to dietetic services
}

\author{
J. D. Youle and J. R. Bebb \\ Derby Hospitals NHS Foundation Trust, Royal Derby Hospital, Uttoxeter Road, Derby, DE22 3NR, UK
}

In 2009, the National Service Standards for $\operatorname{IBD}^{(1)}$ were published, stating that: all patients should have access to a dietitian; patients with Crohn's disease should have access to appropriate liquid enteral nutritional therapy as primary treatment; and where there are alternative treatment options then information and support should be offered to patients for enabling them to participate in decisions about which treatment to select - this might include choice between drug and dietary therapy for Crohn's disease. To explore whether these standards were being upheld locally, a survey was sent to 266 patients on our IBD database to determine: patient awareness of therapeutic diets; whether patients had access to a dietitian; whether patients felt they had nutritional problems; and whether they would like to see a specialist dietitian.

One hundred and thirteen patients responded (43\% response rate), representing views from both IBD sub-types (52\% UC; $44 \%$ Crohn's), both sexes (57\% female; $41 \%$ male) and a wide range of adult age groups.

Seventy-nine percent of patients were not aware of any specific nutritional treatments for IBD. Of those who were aware (19\%, $n 21)$, diets commonly reported were 'low-residue' diets $(n 9)$ or those excluding specific food(s) ( $n$ 16). Only $10 \%$ of the 50 Crohn's respondents were aware of liquid diets.

Thirty-four percent of patients had been offered an appointment with a dietitian in the past, of which $87 \%$ had found it helpful. Eightyfive percent of all respondents would like an appointment with a specialist dietitian to discuss the nutritional management of their IBD. The reasons for wanting to see a dietitian were wide and varied, but the most commonly cited reason was to obtain symptom improvement.

Almost a third of patients $(30 \%)$ did not feel that they knew enough about nutrition to maintain good health and $27 \%$ of patients claimed to have no knowledge about the process of digestion/absorption. Several patients reported weight problems (overweight, underweight or unintentional weight change). Forty percent patients $(n 45)$ had received some nutritional advice to help manage their IBD, mostly regarding low-residue diets, nutritional support or food exclusions. This advice was received from a dietitian (62\%, $n 28)$, IBD nurse ( $n$ 9), consultant $\left(\begin{array}{l}n \\ 7)\end{array}\right)$ or GP $(n$ 2). Others had used magazines, health shops, the internet or books as a source of advice. Forty percent of patients took a range of conventional and unconventional nutritional supplements. Almost a quarter (24\%, $n 27)$ of patients considered themselves to have a food allergy/intolerance. Seventy-nine of them had self-diagnosed their intolerance and $52 \%$ followed a 'special' diet, primarily excluding the 'problem food(s)' 1 . Only $14 \%$ received any professional advice regarding the appropriate diet to follow.

This demonstrates that a significant number of IBD patients have concerns over their diet and make changes without professional advice, which may lead to inappropriate dietary restrictions and compromise nutritional status. It also implies that Crohn's patients are not routinely being informed of the option of exclusive liquid enteral nutrition as primary treatment. Dietetic support is available to patients, but they are unable to self-refer and so may not be aware that they can access services. They are therefore reliant on other health professionals to identify potential nutritional problems and refer accordingly.

Three improvements to practice are proposed: (1) the development of a patient information leaflet for all newly diagnosed IBD patients regarding general nutrition, including information about balanced diets, local nutritional services and how to obtain a referral to a dietitian, (2) the development of a patient information leaflet for all newly diagnosed Crohn's patients outlining the role of liquid enteral nutrition as primary therapy, allowing patients to make informed choices regarding their IBD management, (3) malnutrition screening to be introduced to outpatient areas to identify at risk patients. These measures should improve access to dietetic services, aid compliance with National IBD Standards ${ }^{(1)}$ and importantly, empower patients to safely manage their own nutrition.

1. IBD Standards Group (2009) Quality Care Service Standards for the Healthcare of People who have Inflammatory Bowel Disease (IBD) 